\title{
Community Enterprises - Fremdkörper in der Marktwirtschaft?
}

Edited by: Osterloh, Margit ; Frey, Bruno S ; Lüthi, Roger

Posted at the Zurich Open Repository and Archive, University of Zurich ZORA URL: https://doi.org/10.5167/uzh-90782

Edited Scientific Work

Originally published at:

Community Enterprises - Fremdkörper in der Marktwirtschaft? Edited by: Osterloh, Margit; Frey, Bruno S; Lüthi, Roger (2013). DGRI Jahresbuch 2012: Dr. Matthias Scholz und Dr. Axel Funk. 


\title{
Community Enterprises - Fremdkörper in der Marktwirtschaft ${ }^{1}$
}

\author{
Margit Osterloh/Roger Lüthi/Bruno S. Frey
}
I. Wikipedia als das Musterbeispiel für ein Community Enterprise
II. Eigenschaften von Community Enterprises
1. Welche Ressourcen werden in Community Enterprises produ- ziert?
2. Wie werden Ressourcen in Community Enterprises produ- ziert?
3. Warum werden Ressourcen in Community Enterprises produ- ziert?

III. Community Enterprises als Fremdkörper in der Marktwirtschaft

IV. Implikationen für die Theoriebildung

V. Schlussfolgerungen

Literatur: Amabile, T. M. (1996). Creativity in context: Update to "The Social Psychology of Creativity. “ Westview Press; Baldwin, C., \& Hippel, E. von. (2011). Modeling a Paradigm Shift: From Producer Innovation to User and Open Collaborative Innovation. Organization Science, 22(6), 1399-1417. doi:10.1287/ orsc.1100.0618; Benkler, Y. (2002). Coase's Penguin, or, Linux and „The Nature of the Firm". The Yale Law Journal, 112(3), 369-446. doi:10.2307/1562247; Benkler, Y. (2004). Sharing Nicely: On Shareable Goods and the Emergence of Sharing as a Modality of Economic Production. The Yale Law Journal, 114(2), 273-358. doi:10.2307/4135731; Benkler, Y. (2006). The Wealth of Networks: How Social Production Transforms Markets and Freedom. Yale University Press; Bennis, W. G., \& O’Toole, J. (2005). How Business Schools Lost Their Way. Harvard Business Review, 83(5), 96-104; Bessen, J., \& Meurer, M. J. (2008). Patent Failure: How Judges, Bureaucrats, and Lawyers Put Innovators at Risk. Princeton University Press; Chesbrough, H. (2006). Open Business Models: How To Thrive In The New Innovation Landscape. Harvard Business Press; Corley, K. G., \& Gioia, D. A. (2011). Building Theory about Theory Building: What Constitutes a Theoretical Contribution? Academy of Management Review, 36(1), 12-32; Csikszentmihalyi, M. (1975). Beyond boredom and anxiety: the experience of play in work and games. Jossey-Bass; Dahlander, L. (2007). Penguin in a new suit: a tale of how de novo entrants emerged to harness free and open source software communities. Industrial and Corporate Change, 16(5), 913-943. doi:10.1093/icc/dtm026; Deci, E. L., \& Ryan, R. M. (2000). The "What" and "Why" of Goal Pursuits: Human Needs and the Self-Determination of Behavior. Psychological Inquiry, 11(4), $227-$

1 Gekürzte Version von: Bruno S. Frey, Roger Lüthi und Margit Osterloh, „Community Enterprises- -Gedankenstrich? An Institutional Innovation" in: Managerial and Decision Economics 33 (2012), S. 427-439. 
268. doi:10.1207/S15327965PLI1104_01; Demil, B., \& Lecocq, X. (2006). Neither Market nor Hierarchy nor Network: The Emergence of Bazaar Governance. Organization Studies, 27(10), 1447-1466. doi:10.1177/0170840606067250; Fehr, E., \& Fischbacher, U. (2002). Why Social Preferences Matter - the Impact of Non-Selfish Motives on Competition, Cooperation and Incentives. The Economic Journal, 112(478), C1-C33. doi:10.1111/1468-0297.00027; Frey, B. S. (2008). Happiness: A Revolution in Economics. The MIT Press; Frey, B. S., \& Jegen, R. (2001). Motivation Crowding Theory. Journal of Economic Surveys, 15(5), 589-611. doi:10.1111/1467-6419.00150; Frey, B. S., \& Meier, S. (2004). Pro-social behavior in a natural setting. Journal of Economic Behavior Æ) Organization, 54(1), 65-88. doi:10.1016/j.jebo.2003.10.001; Frey, B. S., \& Stutzer, A. (2002). What Can Economists Learn from Happiness Research? Journal of Economic Literature, 40(2), 402-435; Frischmann, B. (2009). Spillovers Theory and Its Conceptual Boundaries. William and Mary Law Review, 51, 801-824; Frischmann, B., \& Lemley, M. A. (2007). Spillovers. Columbia Law Review, 107(1), 257-301; Frost, J., Osterloh, M., \& Weibel, A. (2010). Governing Knowledge Work: Transactional and Transformational Solutions. Organizational Dynamics, 39(2), 126-136. doi:10.1016/j.orgdyn.2010.01.002; Gächter, S., Krogh, G. von, \& Haefliger, S. (2010). Initiating private-collective innovation: The fragility of knowledge sharing. Research Policy, 39(7), 893-906. doi:10.1016/j.respol.2010.04.010; Gagné, M., \& Deci, E. L. (2005). Self-determination theory and work motivation. Journal of Organizational Behavior, 26(4), 331-362. doi:10.1002/job.322; Hansen, S., Berente, N., \& Lyytinen, K. (2009). Wikipedia, Critical Social Theory, and the Possibility of Rational Discourse. The Information Society, 25(1), 38-59. doi:10.1080/01972240802587562; Hay$e k$, F. A. von. (1975). The Pretence of Knowledge. The Swedish Journal of Economics, 77(4), 433-442. doi:10.2307/3439337; Henkel, J. (2006). Selective revealing in open innovation processes: The case of embedded Linux. Research Policy, 35(7), 953-969. doi:10.1016/j.respol.2006.04.010; Henkel, J., \& Baldwin, C. (2010). Modularity for Value Appropriation - How to Draw the Boundaries of Intellectual Property. SSRN eLibrary. Abgerufen von http://papers.ssrn.com/sol3/papers.cf$\mathrm{m}$ ?abstract_id=1340445; Jeppesen, L. B., \& Lakhani, K. R. (2010). Marginality and Problem-Solving Effectiveness in Broadcast Search. Organization Science, 21(5), 1016-1033. doi:10.1287/orsc.1090.0491; Krogh, G. von, \& Hippel, E. von. (2006). The Promise of Research on Open Source Software. Management Science, 52(7), 975-983. doi:10.1287/mnsc.1060.0560; Lakhani, K. R., \& Wolf, R. G. (2005). Why Hackers Do What They Do: Understanding Motivation and Effort in Free/Open Source Software Projects. In J. Feller, B. Fitzgerald, S. A. Hissam, \& K. R. Lakhani (Hrsg.), Perspectives on Free and Open Source Software (S. 3-21). The MIT Press; Landes, W. M., \& Posner, R. A. (2003). The Economic Structure of Intellectual Property Law. Harvard University Press; Layard, R. (2005). Happiness: Lessons from a new science (Bd. ix). New York, NY, US: Penguin Press; Lee, D., \& Mendelson, H. (2008). Divide and Conquer: Competing with Free Technology Under Network Effects. Production and Operations Management, 17(1), 12-28. doi:10.3401/poms.1070.0005; Lerner, J., \& Tirole, J. (2002). Some Simple Economics of Open Source. The Journal of Industrial Economics, 50(2), 197-234. doi:10.1111/1467-6451.00174; Lindenberg, S. (2001). Intrinsic Motivation in a New Light. Kyklos, 54(2-3), 317-342. doi:10.1111/1467-6435.00156; Lindenberg, S., \& Foss, N. J. (2011). Managing Joint Production Motivation: The Role of Goal 
Framing and Governance Mechanisms. Academy of Management Review, 36(3), 500-525. doi:10.5465/AMR.2011.61031808; Menell, P. S. (2007). The Property Rights Movement's Embrace of Intellectual Property: True Love or Doomed Relationship. Ecology Law Quarterly, 34, 713-754; Müller, C., \& Gurevych, I. (2009). Using Wikipedia and Wiktionary in Domain-Specific Information Retrieval. In C. Peters, T. Deselaers, N. Ferro, J. Gonzalo, G. J. F. Jones, M. Kurimo, T. Mandl, u. a. (Hrsg.), Evaluating Systems for Multilingual and Multimodal Information Access (Bd. 5706, S. 219-226). Springer; Nussbaum, M. C., \& Sen, A. K. (1993). The Quality of Life. Oxford University Press; Osterloh, M., \& Frey, B. S. (2000). Motivation, Knowledge Transfer, and Organizational Forms. Organization Science, 11(5), 538-550. doi:10.1287/orsc.11.5.538.15204; Osterloh, M., \& Rota, S. (2007). Open source software development-Just another case of collective invention? Research Policy, 36(2), 157-171. doi:10.1016/j.respol.2006.10.004; Ostrom, E. (1990). Governing the Commons: The Evolution of Institutions for Collective Action. Cambridge University Press; Ostrom, E. (2010). Beyond Markets and States: Polycentric Governance of Complex Economic Systems. American Economic Review, 100(3), 641-672. doi:10.1257/aer.100.3.641; Ostrom, V., Tiebout, C. M., \& Warren, R. (1961). The Organization of Government in Metropolitan Areas: A Theoretical Inquiry. The American Political Science Review, 55(4), 831-842. doi:10.2307/1952530; Powell, W. W. (1990). Neither market nor hierarchy: Network forms of organization. In B. M. Staw \& L. Cummings (Hrsg.), Research in Organizational Behavior (Bd. 12, S. 295-336). JAI Press; Reichman, J. H., \& Dreyfuss, R. C. (2007). Harmonization without Consensus: Critical Reflections on Drafting a Substantive Patent Law Treaty. Duke Law Journal, 57, 85-130; Rosenzweig, R. (2006). Can History Be Open Source? Wikipedia and the Future of the Past. The Journal of American History, 93(1), 117-146. doi:10.2307/4486062; Rumelt, R. (1984). Towards a Strategic Theory of the Firm. In R. Lamb (Hrsg.), Competitive Strategic Management (S. 556-570). Prentice-Hall; Schreyögg, G., \& Sydow, J. (2010). Organizing for Fluidity? Dilemmas of New Organizational Forms. Organization Science, 21(6), 1251-1262. doi:10.1287/orsc.1100.0561; Schroer, J., \& Hertel, G. (2009). Voluntary Engagement in an Open Web-Based Encyclopedia: Wikipedians and Why They Do It. Media Psychology, 12(1), 96-120. doi:10.1080/15213260802669466; Shah, S. K. (2006). Motivation, Governance, and the Viability of Hybrid Forms in Open Source Software Development. Management Science, 52(7), 1000-1014. doi:10.1287/mnsc.1060.0553; Spinello, R. A. (2003). The future of open source software: Let the market decide. Journal of Information, Communication and Ethics in Society, 1(4), 217-233. doi:10.1108/14779960380000237; Surowiecki, J. (2004). The Wisdom of Crowds: Why the Many Are Smarter Than the Few and How Collective Wisdom Shapes Business, Economies, Societies and Nations. Doubleday; Torvalds, L., \& Diamond, D. (2001). Just for Fun: The Story of an Accidental Revolutionary. HarperCollins; Wernerfelt, B. (1984). A resource-based view of the firm. Strategic Management Journal, 5(2), 171-180. doi:10.1002/smj.4250050207; Williams, S. (2002). Free as in Freedom: Richard Stallman's Crusade for Free Software. O'Reilly; Yang, H.-L., \& Lai, C.-Y. (2010). Motivations of Wikipedia content contributors. Computers in Human Behavior, 26(6), 1377-1383. doi:10.1016/j. chb.2010.04.011; Zenger, T. R., Felin, T., \& Bigelow, L. (2011). Theories of the Firm-Market Boundary. The Academy of Management Annals, 5(1), 89-133. doi: 
10.1080/19416520.2011.590301; Zittrain, J. (2008). The Future of the Internet And How to Stop It. Yale University Press.

\section{Wikipedia als das Musterbeispiel für ein Community Enterprise}

Wer im Internet nach Informationen sucht, landet ziemlich bald bei Wikipedia. Ob man am "Irakkrieg", an der „Finanzkrise", an „Transaktionskosten" oder "Lysergsäurediethylamid“ interessiert ist, WikipediaArtikel sind mit hoher Wahrscheinlichkeit unter den ersten Fundstellen gängiger Internetsuchmaschinen. Marktforscher zählen die Internetenzyklopädie zu den zehn populärsten Websites. Unter den Informationsund Nachrichtenwebsites ist sie die unbestrittene Nummer Eins. Als eine frei verfügbare Enzyklopädie, eine öffentliche Plattform für die Integration von Wissen und eine zentrale Informationsdrehscheibe für aktuelle Ereignisse bietet Wikipedia Leistungen, die es vorher nicht gab, und hat einen deutlichen Effekt auf den Markt für Enzyklopädien.

Wikipedia ist das prominenteste Beispiel einer neuen Form der Unternehmung, die auch Projekte wie Linux, Apache, Eclipse, OpenStreetMap und RepRap umfasst. Wir nennen diese neue Form „Community Enterprise" (CE).

Einige dieser Communitys sind recht groß: rund eintausend Entwickler arbeiten am Linux-Kern und Zehntausende tragen zu Wikipedia bei. ${ }^{2}$

Generell sind CEs private Organisationen, die mit einem neuartigen Produktionsprozess in barrierefreien sozialen Gemeinschaften öffentliche Güter produzieren, gekennzeichnet durch Nicht-Ausschließbarkeit und Nicht-Rivalität im Konsum. CEs tragen zur Wirtschaft bei durch den Nutzwert der produzierten Güter wie z.B. Enzyklopädien, Software oder eine geographische Datenbank. Noch wichtiger ist jedoch, dass CEs weitere Innovationen ermöglichen, indem sie zu einem ökonomischen Ökosystem beitragen, das allen offen steht. Die Gemeinschaft, welche CEs herstellt, organisiert sich selbst, ist polyzentrisch und überlappend. Sie will alle Akteure - einschließlich der Gemeinschaft selbst - daran hindern, Kontrolle über die produzierten Ressourcen zu erlangen.

2 "Linux Kernel Development: How Fast it is Going, Who is Doing It, What They are Doing, and Who is Sponsoring it", http://go.linuxfoundation.org/ who-writes-linux-2012, März 2012. "Wikipedia Statistics“, http://stats. wikimedia.org/EN/TablesWikipediansEditsGt5.htm (besucht: 2012-05-28). 
CEs unterscheiden sich von allen anderen Organisationen, die wir kennen. Sie sind keine Firmen, Märkte oder Netzwerke (Demil \& Lecocq, 2006). Sie sind auch keine selbstorganisierten Allmenden (E. Ostrom, 1990) oder „Open Innovation"-Projekte wie InnoCentive (Jeppesen \& Lakhani, 2010), NineSigma und InnovationXchange. Open Innovation-Projekte stützen sich auf viele lose assoziierte Forscher, doch im Gegensatz zu CEs haben die diese Projekte organisierenden Unternehmen die Kontrolle über die entwickelten Technologien. Hingegen zeichnen sich CEs dadurch aus, dass sie produzierte Ressourcen jedermann frei zur Verfügung stellen, keine Kontrolle ausüben und keine Grenzen zwischen sich und ihre Umgebung ziehen.

Mit diesen Charakteristiken fordern CEs gängige Weisheiten heraus. So dürften sie gemäß der Standardökonomik nicht einmal existieren: Sie produzieren öffentliche Güter ohne zentrale Planung und Kontrolle und haben keine privaten Eigentumsrechte - ein innerhalb der Ökonomik nicht zu erklärender Sachverhalt. CEs zeigen jedoch, dass manche Ressourcen genau dann am produktivsten sind, wenn mit ihnen keine Eigentumsrechte verbunden sind. Zudem sind CEs Fremdkörper in der ökonomischen Theorie der Firma. Sie passen nicht zur zentralen Frage dieser Theorie: Was bestimmt die Grenzen der Firma? Daher werden CEs in der Literatur zur Theorie der Firma weitgehend ignoriert (z. B. Zenger, Felin, \& Bigelow, 2011).

Zwar sind Community Enterprises Gegenstand publizierter Forschung, beispielsweise in der Informatik (Hansen, Berente, \& Lyytinen, 2009; Müller \& Gurevych, 2009), in der juristischen Literatur (Benkler, 2002), in der Geschichte der Technik (Rosenzweig, 2006), in der Managementund Innovationsforschung (Osterloh \& Rota, 2007) sowie in der Ökonomik (Lerner \& Tirole, 2002). Jedoch werden CEs meistens nur aus der Perspektive profitorientierter Firmen analysiert. Typische Forschungsinteressen sind Wettbewerbsvorteile von Firmen (z. B. Gächter, Krogh, \& Haefliger, 2010; Krogh \& Hippel, 2006); die Bedingungen, unter denen es für Firmen sinnvoll ist, mit CEs zu kooperieren (Dahlander, 2007; Henkel, 2006); was Firmen von CEs lernen können (Baldwin \& Hippel, 2011); und wie Firmen auf Konflikte mit CEs reagieren können (Lee \& Mendelson, 2008). Ein weitaus kleinerer Teil der Literatur untersucht CEs und ähnliche Kooperationsformen als eigenen Gegenstand. So beschreibt Benkler (2004) die ",commons-based peer production" als eine Kooperation, in der niemand exklusive Rechte erwirbt, um die Arbeit zu kontrollieren oder sich ihren Wert anzueignen. Wir tragen zum zweiten Literaturstrom bei, indem wir analysieren, wie CEs als Fremdkörper in der 
Marktwirtschaft unweigerlich zu Konflikten mit Firmen sowie der gängigen ökonomischen Lehre führen. Zwar profitieren Firmen, Konsumenten und die Öffentlichkeit stark von CEs, doch der Wert der von CEs produzierten Leistungen ist schlecht messbar. Sie gehen nicht in Gewinne, das Bruttoinlandsprodukt oder in Beschäftigungsquoten ein. Im Gegensatz zu vom Staat produzierten öffentlichen Gütern können die von CEs produzierten Leistungen nicht einmal über die Kosten von Inputfaktoren gemessen werden, weil sie entweder von Freiwilligen hergestellt werden oder von Firmen, welche die betreffenden Zahlen nicht veröffentlichen. Leider wird in den Sozialwissenschaften oft nur das als wichtig behandelt, was für Messungen leicht zugänglich ist (Hayek, 1975, S. 434). Auch in der Managementforschung werden üblicherweise nur die Variablen berücksichtigt, die leicht messbar sind. Dies führt zum Paradox, dass neuartige Phänomene eher verborgen als beleuchtet werden. ${ }^{3}$ Das ist ein gewichtiger Nachteil für CEs sowohl als Forschungsthema als auch im politischen Prozess.

Mit diesem Papier tragen wir zur Analyse von CEs als Fremdkörper in der Marktwirtschaft bei. Wir beginnen mit einer Analyse der Schlüsseleigenschaften von CEs (Abschnitt 2). Wir zeigen dann, wie Firmen von CEs profitieren, diese aber nicht unterstützen, sondern deren Prinzipien sogar bekämpfen (Abschnitt 3). Schließlich diskutieren wir Implikationen für die Theoriebildung. Wir kommen zum Schluss, dass die dominanten ökonomischen Theorien die Erforschung dieses neuen Phänomens behindern, obwohl es enorme ökonomische Werte schafft.

\section{Eigenschaften von Community Enterprises}

Community Enterprises unterscheiden sich von traditionellen Firmen darin, was, wie und warum Ressourcen produziert werden. CEs sind Benklers (2006) „,commons-based peer production“ verwandt. Der Fokus unserer Analyse liegt jedoch nicht auf einem Produktionsmodus, sondern auf Unternehmen mit einem Zweck und einer Identität, die zwar einerseits Unternehmen sind, aber nach anderen Prinzipien als profitorientierte Unternehmen funktionieren.

\section{Welche Ressourcen werden in Community Enterprises produziert?}

Community Enterprises zeichnen sich aus durch eine besondere Art von Produkten, welche gleichzeitig Ressourcen darstellen. Sie produzieren

3 Bennis \& O’Toole (2005); Corley \& Gioia (2011). 
öffentliche Güter - Informationsgüter und gleichzeitig Innovationsressourcen -, die jedermann zur freien Verfügung stehen.

Die von CEs produzierten Informationsgüter und Ressourcen kennen keine Rivalität im Konsum und keine Ausschließbarkeit. Grundsätzlich ermöglichen Gesetze, Informationsgüter und Ressourcen ausschließbar zu machen, z.B. durch Urheberrechte. CEs bieten jedoch aus Prinzip einen barrierefreien Zugriff an und entscheiden sich bewusst, ihre Innovationsressourcen in öffentliche Güter zu verwandeln. Offene Standardlizenzen (z.B. Copyleft-Lizenzen wie die GNU General Public License) geben diesem Prinzip eine konkrete Form. Am bekanntesten ist ihre Verwendung in Free and Open Source Software (FOSS), aber bestehende Lizenzen decken auch Werke der Kultur, Datenbanken und Hardware-Konstruktionspläne ab.

Der augenfälligste Aspekt von CEs sind kostenlose Produkte. Wichtiger ist jedoch, dass CEs nicht nur für Konsumenten, sondern auch für Produzenten produzieren. Erstens schaffen CEs Innovationsinfrastruktur wie Softwarebibliotheken, Werkzeuge und Datenbanken. Zweitens sind diese Innovationsressourcen typischerweise außergewöhnlich flexibel. Beispielsweise unterstützt CE-Software zahlreiche Hardwarearchitekturen, Softwareplattformen, Kommunikationsprotokolle und Dateiformate. Drittens stellen CEs ihre Ressourcen in einer Form zur Verfügung, die sie für Nachfolgeinnovatoren und potentielle Konkurrenten besonders attraktiv macht. Sie bieten nicht nur Software an, sondern auch den Quellcode, aus dem diese hergestellt wurde. Sie stellen nicht nur kostenlos enzyklopädische Artikel und Straßenkarten zur Verfügung, sondern auch den Inhalt der Datenbanken und die Software, welche für die Nutzung und Weiterentwicklung gebraucht wird. Das Wikipedia-Projekt beispielsweise verteilt nicht nur enzyklopädische Inhalte, sondern auch eine Wiki-Software, die eigens für Wikipedia entwickelt wurde. Diese Besonderheiten von CE-Leistungen sind nicht nur für Endbenutzer attraktiv, sondern auch für jene, welche die Ressource für ein Produkt anpassen oder ihre zukünftige Entwicklung beeinflussen wollen. Die von CEs angebotenen Produkte sind „generativ“ (Zittrain, 2008), d.h. sie sind vielseitige Allzwecktechnologien und Ressourcen, die frei verwendet und umgenutzt werden können.

\section{Wie werden Ressourcen in Community Enterprises produziert?}

Community Enterprises produzieren polyzentrisch und barrierefrei. Polyzentrische Governance ist charakterisiert durch viele Entscheidungszentren, die voneinander formal unabhängig sind (V. Ostrom, Tiebout, \& 
Warren, 1961). Kein Akteur ist in der Lage, die Entwicklung zu kontrollieren, indem er die Verwendung, die Anpassung oder die Erweiterung der Ressource verbietet. Innerhalb von CEs mögen zwar einige Individuen oder Gruppen beträchtlichen Einfluss auf ein Projekt haben, doch dies nur so lange, wie die anderen Akteure ihre Entscheide freiwillig respektieren. Informelle wohlwollende Diktatoren (z. B. Linus Torvalds beim Linux-Kern) oder formal gewählte Organe (z. B. in den Dachorganisationen von Wikipedia und Apache) sind nicht für zentrale Planung und Kontrolle zuständig, sondern erleichtern vor allem die Kooperation, Koordination und Konfliktlösung zwischen einer Vielzahl autonomer und unabhängiger Gruppen.

Die polyzentrische Governance hat die Barrierefreiheit zur Voraussetzung. Offene Lizenzen geben jedermann die Erlaubnis, die Ressource für eigene Bedürfnisse einzusetzen und zu verbessern. Eine zentrale Befehlsstruktur und der Ausschluss abweichender Ansichten sind daher nicht möglich. Weil CEs keine Kontrolle über ihre Ressourcen sichern müssen, brauchen sie auch keine Grenzen, welche die Unternehmung vom Rest der Welt trennen. Sie bieten einen barrierefreien Zugang zu den Ressourcen und zu den Werkzeugen und Prozessen, mit denen sie geschaffen werden. Lose Strukturen und informale Prozesse sind typisch für diese Art von Unternehmung.

Community Enterprises erfüllen zugleich die vier Bedingungen für kollektive Intelligenz oder „wisdom of crowds" (Surowiecki, 2004): Diversität, Unabhängigkeit, Dezentralisierung und Aggregation. Erstens ist die Diversität an Meinungen und fachlichem Hintergrund unter den Mitgliedern von CEs bemerkenswert hoch. Es gibt keine Barrieren bezüglich formaler Qualifikation, Herkunft oder Firmenzugehörigkeit. Zweitens bleiben die Mitglieder von CEs unabhängig. Von ihnen wird nicht erwartet, dass sie für eine bestimmte (oder überhaupt eine) Firma arbeiten oder bestimmte Ansichten äußern. Die Diversität der Herkunftsorte und Zielvorstellungen ist ebenfalls groß. Drittens sind die Entscheidungsprozesse in CEs dezentral. Gruppen und Individuen erweitern die CE-Ressource nach eigenem Ermessen auf der Basis lokalen Wissens sowie eigener Perspektiven und Interessen. Die Koordination mit anderen ist keine Pflicht, sondern freiwillig. Viertens verfügen CEs über Mechanismen, welche es ihnen erlauben, Informationen und Beiträge von vielen Akteuren zu aggregieren. Ein prominentes Beispiel ist die Wiki-Software, die es vielen Autoren gleichzeitig erlaubt, kollaborativ Texte zu editieren.

Es ist erstaunlich, dass CEs trotz des Fehlens von Grenzen, Hierarchie und Kontrolle und trotz der hohen Diversität soziale Gemeinschaften 
mit oft stark entwickelten Identitäten darstellen. Dies steht im Gegensatz zur traditionellen Organisationstheorie, welche definierte Grenzen als wichtigste Voraussetzung für Identität betrachtet (z. B. Schreyögg \& Sydow, 2010). Trotz der Heterogenität und der losen Strukturen wird in CEs Identität vermittelt durch die Natur der geschaffenen Ressource, die Projektgeschichte und -kultur sowie durch die laufenden Interaktionen zwischen den am Projekt beteiligten Individuen. So trägt das Ziel, eine freie Enzyklopädie herzustellen, ebenso zur gemeinsamen Identität bei wie Diskussionen auf projektbezogenen Webseiten und Mailinglisten. Die Zugehörigkeit zu CEs wird daher nicht durch Grenzen bestimmt, sondern einerseits durch Interaktionen mit der gemeinsamen Ressource und andererseits durch das gegenseitige Verhalten. Es gibt in der Regel keine formalen Mitglieder, sondern die Akteure assoziieren sich mit dem CE durch die Verwendung der Ressource, die Teilnahme an Diskussionen, Werbung für das Projekt oder Beiträge zur Weiterentwicklung. In einem CE erwerben Individuen Zugehörigkeit einfach dadurch, dass sie entsprechend agieren und interagieren.

Die Art und Weise, wie Ressourcen in CEs produziert werden, ist relevant für ein zentrales Problem von Organisationen: das Agenturproblem. Traditionelle Organisationen versuchen die Kontrolle über zentrale Ressourcen zu gewinnen, welche ihnen Wettbewerbsvorteile verschaffen (Zenger u. a., 2011). Sind sie erfolgreich, wächst nicht nur der Wert der Aktiven einer Organisation, sondern auch der potentielle Gewinn opportunistischen Verhaltens. Um die Veruntreuung wertvoller Aktiven zu verhindern, sind machtvolle Governancesysteme nötig. Im Gegensatz dazu sind CEs weitgehend immun gegen Agenturprobleme und können daher mit informellen Governancestrukturen operieren.

Zusammenfassend halten wir fest, dass CEs die typischen Instrumente von Firmen - zentrale Planung und Kontrolle - mit einer polyzentrischen, überlappenden Governance ersetzen. CEs bieten keine Anreize für die Umsetzung eines zentralen Plans. Stattdessen bieten sie jedermann Möglichkeiten und Werkzeuge, um an und mit der Ressource zu arbeiten. Barrierefreier Zugang und polyzentrische Governance schaffen die Voraussetzungen für kollektive Intelligenz und eine breite Auswahl von Motiven. CEs bieten barrierefreien Zugang zu Produktionsprozessen und Ressourcen, die üblicherweise aus Gründen des Wettbewerbs und der Effizienz von Organisationen nicht frei zugänglich sind. 


\section{Warum werden Ressourcen in Community Enterprises produziert?}

Im Gegensatz zu profitorientierten Unternehmungen haben CEs nicht das Ziel, sich den Nutzen der produzierten Ressourcen anzueignen, sondern diese frei zugänglich $\mathrm{zu}$ machen. Die Institutionen und Prozesse von CEs sollen alle - auch die Schöpfer der Ressource selbst - daran hindern, Kontrolle über die Verwendung und die weitere Entwicklung der Ressource zu erlangen. Daher ist Kontrolle kein möglicher Anreiz für die Schaffung der Ressource. Es stellt sich die Frage, aus welchen Gründen Ressourcen in CEs hergestellt werden. Einerseits ist zu klären, warum Individuen ohne externe Anreize Beiträge an CEs leisten. Andererseits muss es Qualitäten geben, welche die Produkte von CEs für die Nachfrageseite attraktiv machen.

Individuen beteiligen sich aus drei verschiedenen Motivationen an den Aktivitäten von CEs. Diese schließen einander nicht aus, sondern können in unterschiedlichem Ausmaß aktiviert werden (Lindenberg, 2001; Lindenberg \& Foss, 2011; Osterloh \& Frey, 2000). Es sind dies extrinsische Motivation, hedonistisch-intrinsische und pflichtbewusst-intrinsische Motivation. ${ }^{4}$ Extrinsische Motivation bezeichnet eine Aktivität, welche auf ein Resultat, etwa Geld und materielle Belohnungen gerichtet ist (Deci \& Ryan, 2000). Intrinsische Motivation basiert dagegen auf der Befriedigung, die eine Aktivität ohne externe Belohnungen gewährt. Hedonistisch-intrinsische Motivation verursacht ein befriedigendes „Flow"-Erlebnis einer Aktivität (Csikszentmihalyi, 1975), beispielsweise beim Spielen oder beim Lösen interessanter Probleme. Diese Art Motivation tritt häufig in der Forschung (Amabile, 1996) oder während der Programmierung von Software auf (Torvalds \& Diamond, 2001). In solchen Fällen ist die Aktivität selbst mit Vergnügen verbunden. Pflichtbewusst-intrinsische Motivation bezeichnet Aktivitäten, deren Zweck angemessenes Verhalten ist. Wenn Individuen eine solche Motivation haben, folgen sie Normen aus einem prosozialen Anliegen. Insbesondere beziehen sie das Wohlergehen anderer in ihre Entscheidungen ein, ohne eine Belohnung zu erwarten. Das Wohlergehen der Gemeinschaft wird Teil individueller Präferenzen.

Während das ökonomische Standardmodell menschlichen Verhaltens, der Homo Oeconomicus, auf der Annahme eigennütziger, extrinsisch

4 Wir ziehen das Konzept unterschiedlicher motivationaler Frames aus Lindenberg (2001) und Lindenberg und Foss (2011) dem Konzept eines Motivationskontinuums von Gagné und Deci (2005) vor, weil es besser zur Frage passt, wie Individuen in einem Team zu motivieren sind. 
motivierter Individuen basiert, gibt es zahlreiche empirische Evidenz dafür, dass viele Leute freiwillig zu Gemeinschaften beitragen, zu denen sie sich zugehörig fühlen (z. B. Fehr \& Fischbacher, 2002; Frey \& Jegen, 2001; Frey \& Meier, 2004; Frost, Osterloh, \& Weibel, 2010).

Alle drei Arten von Motivation finden sich in CEs. Viele Beiträge zu CEs sind auf Eigenbedarf oder extrinsische Belohnungen wie Bezahlung, Reputation oder Weiterbildung zurückzuführen. Beispielsweise wird die Entwicklung des Linux-Kerns mehrheitlich von bezahlten Entwicklern erledigt. Shah (2006) ermittelte, dass Verbesserungen bestehender CE-Software oft durch eigene Bedürfnisse angetrieben wird; die Entwickler "scratch their own itch", wie es oft umschrieben wird. Hedonistisch-intrinsische Motivation ist schon im Titel von Torvalds und Diamond (2001) offenkundig: "Just For Fun". Lakhani und Wolf (2005) identifizieren als wichtigsten Treiber das Bestreben nach Kreativität. Pflichtbewusst- intrinsische Motivation findet sich ebenfalls häufig in CEs. Auch sie ist in den Biographien bedeutender Figuren wie Richard Stallman (Williams, 2002) und in Umfragen klar erkennbar. Viele lassen sich von prosozialen Selbstkonzepten leiten, wenn sie Informationen weitergeben (Yang \& Lai, 2010). Stark engagierte Wikipedia-Editoren sehen oft wenig individuellen Nutzen in ihrer Arbeit, haben aber ein Interesse daran, Informationen weiterzugeben oder den Wunsch, künftigen Generationen ein positives Erbe zu hinterlassen (Schroer \& Hertel, 2009).

Wikipedia, Linux, OpenStreetMap und viele andere erfolgreiche CEs haben ihr anfängliches Wachstum mit intrinsisch motivierten Beiträgen erzielt, bis sie eine kritische Größe erreicht hatten. Zu Beginn waren die Projekte Experimente. Sie zogen Enthusiasten an. Extrinsische Motivationen wurden erst prominent, nachdem CEs funktionierende Prozesse etabliert und wichtige Ressourcen geschaffen hatten (Osterloh \& Rota, 2007).

Auf der Nachfrageseite sind die Produkte von CEs attraktiv, weil sie Innovationsressourcen schaffen, welche für deren Nutzung und Weiterverwendung frei verfügbar sind und bleiben. Nachfolgeinnovatoren, die in eine CE-Ressource investieren, werden dadurch nicht abhängig, weil die Ressource von niemandem kontrolliert wird. Kein anderer Investor kann die Firma daran hindern, die Ressource ihren Bedürfnissen entsprechend zu verwenden. Die generativen Technologien von CEs können nicht zurückgezogen werden.

Darüber hinaus verstärken Community Enterprises die Nachfrage nach weiteren frei verfügbaren Innovationsressourcen. Sie benötigen solche 
Ressourcen viel dringender als Firmen. Firmen können beispielsweise teure Softwarewerkzeuge und andere Innovationsressourcen kaufen, während CEs Alternativen finden müssen, wollen sie Freiwillige anziehen. Diese Alternativen sind oft nur erhältlich, wenn sie von anderen CEs produziert werden. Umgekehrt liefern bestehende CEs oft die Inspiration und die generativen Technologien für neue CE-Projekte. So gelang die Entwicklung von Linux auf der Grundlage bestehender, offen lizenzierter Softwarewerkzeuge. Wikipedia verwendet wiederum Linux und andere, offen lizenzierte Software. Die Existenz von CEs in einigen Gebieten schafft also eine Nachfrage für die Ausdehnung von CEs in andere Gebiete. Ein selbstverstärkender Kreislauf setzt ein.

Zusammenfassend lässt sich sagen, dass auf der Angebotsseite eine Mischung unterschiedlicher Motivationen für individuelle Beiträge zu CEs sorgt. Auf der Nachfrageseite befriedigen CEs einen Bedarf nach Technologien und Innovationsressourcen, die dauerhaft ohne Zugangsbeschränkungen verwendet und verbessert werden können.

\section{Community Enterprises als Fremdkörper in der Marktwirt- schaft}

Community Enterprises sehen sich einzigartigen Herausforderungen gegenüber, weil sie unbeschränkt Zugang gewähren zu Ressourcen, die sie produzieren.

Diese Herausforderungen entstehen aus Interaktionen mit einer ökonomischen und juristischen Umwelt, die CEs nicht nur zu Fremdkörpern in der Marktwirtschaft machen, sondern auch - wenigstens teilweise zu starken Konkurrenten. In diesem Abschnitt analysieren wir die Interaktionen zwischen CEs und Firmen, welche sowohl Herausforderungen als auch neue Möglichkeiten schaffen. Einerseits profitieren Firmen von CEs und geben ihnen auch manchmal Unterstützung. Andererseits sind diese Akteure wenig verlässliche Verbündete, weil ihre Unterstützung begrenzt ist und weil sie CEs oft als Konkurrenten bekämpfen. Obwohl CEs viel zur Gesamtwirtschaft beitragen, leiden sie darunter, dass sich ihre Beiträge mit den gängigen ökonomischen Instrumenten nicht erfassen lassen. Politische Entscheidungen neigen in der Folge dazu, die Interessen von CEs zu ignorieren oder nicht ernst zu nehmen, was den Umfang und die Qualität der Ressourcen einschränkt, welche CEs produzieren können. 
Firmen haben bei der Schaffung und Entwicklung von CEs eine entscheidende Rolle gespielt - sowohl als Verbündete als auch als Gegner. Sie gehören zu den Hauptprofiteuren der von CEs produzierten Ressourcen. Startup-Unternehmen verwenden bevorzugt offen lizenzierte, generative Technologien, um ihr Geschäft schnell und zu geringen Kosten aufzubauen. Ehemalige Startup-Unternehmen wie Yahoo, Google und Facebook setzen offene Ressourcen in unternehmenskritischen Bereichen ein, auch lange nachdem sie groß geworden sind. Der Reiz von FOSS ist nicht auf Internetdienste beschränkt. Auch Hersteller von Netzwerkgeräten und Konsumelektronik verwenden FOSS. Sogar Börsenplätze verlassen sich für Hochleistungstransaktionsverarbeitung zunehmend auf FOSS. ${ }^{5}$ Firmen haben die von CEs geschaffenen frei verfügbaren Ressourcen schätzen gelernt und unterstützen ausgewählte Projekte. Das wohl prominenteste Beispiel ist der Linux-Kern. Zu seinen Sponsoren zählen viele der größten Produzenten von Software, Halbleitern, Konsumelektronik und Internetdiensten. Mit seinem jährlichen "Summer of Code"-Programm hat Google Tausenden von Studenten Stipendien gewährt, um an Hunderten von FOSS-Projekten mitzuarbeiten. Sogar die Dachorganisation von Wikipedia, die besonders darauf achten muss, jeglichen Anschein von Parteilichkeit oder Käuflichkeit zu vermeiden, hat Firmensponsoren. ${ }^{6}$

Firmen sind jedoch wenig verlässliche Verbündete für CEs, denn ihre Interessen kollidieren oft, insbesondere in Bezug auf die Exklusivität und Aneignung von Ressourcen, auf die Governance, auf Wettbewerbsstrategien und vor allem in Bezug auf Regulierungsaspekte.

Exklusivität und Aneignung von Ressourcen. Die Aneignung von Ressourcen - durch Eigentum oder durch andere Formen exklusiver Kontrolle - ist ein Mittel zur Erlangung von Wettbewerbsvorteilen. Gemäß dem Resource-Based View der Strategielehre müssen diese Ressourcen wertvoll, selten, schwer nachzuahmen und nichtsubstituierbar sein. Firmen versuchen daher, ihre Ressourcen durch Isolationsmechanismen (Rumelt, 1984) oder Barrieren (Wernerfelt, 1984) zu schützen. Solche Mechanismen und Barrieren wurden vor allem durch die Ausdehnung geistiger Eigentumsrechte geschaffen und gestärkt. Viele Firmen haben in der Folge Geschäftsmodelle entwickelt, die sowohl starke als auch schwache

5 Leo King, „London Stock Exchange Linux record breaking system faces new challengers" , http://www.computerworlduk.com/in-depth/open-source/3246835/ (2010-11-02).

6 "Benefactors", http://wikimediafoundation.org/w/index.php?title=Benefactors \&oldid $=58505$. 
Aneignungsregimes verwenden (Chesbrough, 2006). Diese verursachen oft Konflikte mit CEs. Beispielsweise kooperierte AT\&T zu Beginn der Unix-Entwicklung mit anderen Firmen und Universitäten. Nach der Aufteilung der Firma von 1984 versuchte AT\&T, die Lizenzeinnahmen für Unix zu steigern. Als Reaktion darauf ersetzte die Gemeinschaft die Teile von Unix, die Eigentum von AT\&T waren. So wurde ein freies Unix geschaffen, für das keine Lizenzgebühren mehr fällig waren.

Weil CE-Ressourcen für profitorientierte Firmen einen großen Nutzen haben, wollen sie eine exklusive Kontrolle über deren Verwendung erlangen. Das aber widerspricht dem Ziel von CEs und ruft Widerstand hervor. Entsprechende Maßnahmen haben oft weitreichende Konsequenzen, weil sie in der Regel Lizenzierungsangelegenheiten betreffen, die auch für viele andere CEs relevant sind. Beispielsweise war die Copyleft-Klausel eine Reaktion auf Firmen, welche offene Ressourcen verwendeten, aber Verbesserungen als proprietäre Software verkauften (z. B. Osterloh \& Rota, 2007).

Governance. Firmen appropriieren Wert, indem sie kollektives Handeln durch Planung und Kontrolle steuern. Im Gegensatz dazu schaffen CEs nicht nur bewusst Ressourcen in Form von öffentlichen Gütern, sondern verhindern genau die Art von Kontrolle über Ressourcen, welche Firmen benötigen. Sie weisen die Idee exklusiver Rechte an der Ressource zurück und damit auch die Mittel, die den Governancestrukturen von profitorientierten Firmen zugrunde liegen.

Wettbewerbsstrategien. CEs sind für Firmen Konkurrenten, welche die gängigen Regeln des Wettbewerbs in der Marktwirtschaft bedrohen. Erstens stellen CEs unbegrenzte Mengen ihrer Ressource kostenlos zur Verfügung. Sie stellen damit eine andere Form der Konkurrenz dar als neu eintretende Firmen, welche zwar mit tiefen Preisen Marktanteile gewinnen wollen, aber dennoch Interesse an gewinnbringenden Margen haben.

Zweitens können CEs nicht gekauft werden. Was sie produzieren, sind keine Aktiven, sondern offen lizenzierte und daher unwiderruflich für jedermann frei zugängliche Ressourcen. Einige wenige Rechte - etwa Trademarks - gehören oft einer eigens geschaffenen, gemeinnützigen Organisation; selbst wenn eine Firma in den Besitz solcher Namensrechte käme, würde die offene Lizenz es anderen erlauben, die Ressource zu kopieren und unter einem neuen Namen weiterzuführen. Das ist bei der Übernahme von Sun Microsystems durch Oracle geschehen. Mit dieser Transaktion erwarb Oracle auch die Namensrechte mehrerer FOSS-Projekte, darunter OpenOffice.org und Hudson. Die meisten Unterstützer 
von OpenOffice.org verließen jedoch das Projekt und führten ihre Arbeit an den Büroprogrammen unter dem neuen Namen LibreOffice fort. Die Community, welche die Integrationssoftware Hudson entwickelte, reagierte ebenfalls negativ. Sie gab den Namen, den Oracle für sich beanspruchte, auf und gründete ein neues CE unter dem Namen Jenkins.

Drittens erhöhen CEs den Konkurrenzdruck in angrenzenden Feldern. Wenn CEs die Nachfrage nach - und die Möglichkeiten für - weitere CE-Projekte schaffen, dehnen sie sich in zusätzliche Felder aus. Weil Firmen den Effekt freier Ressourcen in der Regel nur dann schätzen, wenn das den Wert der von ihnen kontrollierten Ressourcen erhöht (vgl. Henkel \& Baldwin, 2010), begrüßen sie solche Ausdehnungen von CEs nicht.

Regulierung. Dies ist das wichtigste Schlachtfeld zwischen Firmen und CEs. CEs sind sich beispielsweise einig in der Ablehnung von Softwarepatenten, während Industrielobbyisten solche Patente unterstützen. ${ }^{7} \mathrm{~Pa}-$ tente erlauben es Firmen, Kontrolle und Eigentumsrechte über Software auszuüben, selbst wenn die Urheberrechte offen lizenziert sind. Eng verwandt mit Softwarepatenten ist die laufende Debatte über die Definition offener Standards. Auf der einen Seite argumentieren CEs, dass die Verwendung von Standards weder eine Genehmigung noch Lizenzzahlungen erfordern darf. Regierungen in aller Welt haben dieses Argument unterstützt, indem sie Schritte unternommen haben, um in ihren Beschaffungsrichtlinien offene Standards zu bevorzugen. Auf der anderen Seite argumentieren Industrielobbyisten, die Förderung offen lizenzierter Produkte und lizenzkostenfreier Standards sei unangebracht; die Regierungen sollten stattdessen „den Markt entscheiden lassen" (vgl. Spinello, 2003). Aus dieser Sicht sollten Regierungen, welche die Beiträge von CEs beurteilen, sich wie profitorientierte Firmen verhalten und die Vorteile für die öffentliche Wohlfahrt ignorieren.

Die bisherige Analyse zeigt, dass Firmen die Methoden und Praktiken von CEs nicht übernehmen können, ohne die Kontrolle zu verlieren, welche ihre Geschäftsmodelle lukrativ macht. Sie sind deshalb bestrebt, Regulierungen zu schaffen, welche es ihnen erlauben, Kontrolle auszubauen oder den Wert der von CEs hergestellten Ressourcen für die Öffentlichkeit zu schwächen. Daher gibt es von Firmen wenig Unterstützung für CEs. Tun sie es dennoch, dann nur insoweit, als das ihre Kontrolle über wettbewerbsrelevante Ressourcen stärkt.

$7 \mathrm{Zu}$ den Unterstützern von Softwarepatenten gehören viele grosse Firmensponsoren von FOSS-Projekten. 


\section{Implikationen für die Theoriebildung}

In diesem Abschnitt diskutieren wir die theoretischen Implikationen dieser Entwicklungen für die Standardökonomik und für die Theorie der Firma, die auf der Standardökonomik basiert. Wir untersuchen deren Annahmen, welche eine adäquate Analyse von CEs behindern und deren Potential als Quelle institutioneller Innovation und ökonomischen Nutzens nicht erfassen können.

In der Ökonomik werden erstens Externalitäten üblicherweise als Problem betrachtet. Entsprechend ist die Internalisierung von externen Effekten das Standardrezept der Ökonomik zur Behandlung von externen Effekten. Geistige Eigentumsrechte sind ein prominentes Beispiel dafür. Indem sie öffentliche Güter ausschließbar machen, ermöglichen sie Investoren die Aneignung von Nutzen, der sonst eine positive Externalität ihrer Arbeit wäre. Die Existenz von CEs zeigt jedoch, dass in manchen Fällen die Internalisierung externer Effekte weniger vorteilhaft ist als die Erhaltung der Nichtausschließbarkeit eines öffentlichen Gutes. Zu diesem Schluss gelangt auch die Spillover-Theorie (Frischmann, 2009; Frischmann \& Lemley, 2007), die nicht als Teil der Standardökonomik gilt.

Eine zweite Annahme favorisiert starke Eigentumsrechte und dehnt diese Sicht auf geistiges Eigentum aus. Dies steht im Gegensatz zu empirischer Evidenz, welche keineswegs den gesamtwirtschaftlichen Nutzen geistigen Eigentums bestätigt (z. B. Bessen \& Meurer, 2008; Landes \& Posner, 2003; Menell, 2007). Die gut dokumentierten Vorteile des Privateigentums an materiellen Gütern gelten nicht automatisch für immaterielle Güter. Das zeigt auch die Tatsache, dass CEs Wert schaffen, indem sie bewusst Ressourcen produzieren, die niemandem gehören.

Eine dritte Annahme betrifft die Natur von Innovation. Seit Joseph Schumpeter gelten produzierende Firmen als Hauptquelle von Innovation (Baldwin \& Hippel, 2011). Diese Annahme stimmt nicht für CEs. Das Internet basiert auf nicht-proprietären Technologien und wurde zum Treibhaus für kommerzielle und nichtkommerzielle Innovation, indem es jedermann erlaubte, sich am Wettbewerb um Aufmerksamkeit und Kaufkraft zu beteiligen. Die Rolle solcher Innovationsmotoren lässt sich im Rahmen der Standardökonomik nicht beschreiben.

Viertens kann eine Theorie der Firma, welche sich auf die Frage nach den Grenzen der Firma konzentriert, die Eigenschaften von barrierefreien Unternehmungen - wie den CEs - nicht erfassen. Das ist vor allem deswegen problematisch, weil in den meisten Formulierungen der Theorie 
die Grenzen der Firma anhand der Reichweite der Autorität zentraler Entscheidungsträger bestimmt werden. In CEs gibt es keine solche Autorität. Das könnte erklären, warum Übersichten zur Theorie der Firma die Existenz von CEs und ähnlichen Gemeinschaften nicht einmal erwähnen (z. B. Zenger u. a., 2011).

Unpassende Annahmen stellen für die Erforschung neuer Phänomene große Hürden dar. Erstens werden neue Phänomene wie CEs im Licht konventioneller Theorien unterschätzt. Das ist insbesondere darauf zurückzuführen, dass konventionelle Maße für ökonomische Aktivitäten - etwa BIP, Anzahl Beschäftigter, Gewinne, F\&E-Ausgaben oder Patentaktivitäten - den Nutzen von CEs für die Gesamtwirtschaft fast vollständig ausblenden. Mögliche alternative Maße, welche CEs einschließen, wären etwa die Messung von Glück (Frey, 2008; Frey \& Stutzer, 2002; Layard, 2005) oder von Capabilities (Nussbaum \& Sen, 1993).

Zweitens ist es nicht möglich, theoriegeleitet Voraussetzungen für die Entstehung von CEs zu identifizieren. Dies wäre erst dann der Fall, wenn die ökonomische Theorie der Firma zu einer umfassenden Theorie der Unternehmung erweitert würde, welche neben Firmen auch CEs umfasst.

Drittens sind CEs in konventionellen empirischen Studien in der Regel nicht Teil der Stichproben. Dadurch werden Mängel bestehender Theorien nicht bemerkt, welche CEs betreffen.

Viertens erschwert die internationale Harmonisierung von Gesetzen empirische Studien. Die abnehmende Vielfalt der juristischen Umwelt eliminiert Möglichkeiten, die Auswirkungen unterschiedlicher Regulierungen zu erforschen (Reichman \& Dreyfuss, 2007).

Fünftens werden Gesetzesänderungen diskutiert, welche die existierenden Nischen für CEs weiter einschränken oder ganz zerstören könnten, z.B. die Verlängerung von Schutzfristen und ein Leistungsschutzrecht für Verleger.

Zur tieferen Analyse dieser Probleme wäre allerdings eine umfassende Theorie der Unternehmung nötig, welche sowohl die Existenz von Firmen als auch die Existenz von CEs erklärt und daraus Hinweise für ein konfliktärmeres Einpassen von CEs in die Marktwirtschaft ableitet. 


\section{Schlussfolgerungen}

Die ökonomische Forschung hat sich lange auf das Studium von profitorientierten Organisationen konzentriert, welche basierend auf zentraler Autorität und innerhalb klar definierter Grenzen Ressourcen im Privateigentum produzieren. Andere Arten von Unternehmungen haben zwar in den vergangenen Jahrzehnten ebenfalls einige Prominenz erlangt. So hat die Literatur zu Allmenden gezeigt, dass Ressourcen im Kollektiveigentum mit selbstorganisierter, polyzentrischer Governance erfolgreich verwaltet werden können (E. Ostrom, 1990, 2010; Powell, 1990). Aber diese Unternehmungen sind exklusive Klubs, die auf starke persönliche Beziehungen oder klar definierte Grenzen angewiesen sind. Sie kontrollieren physische, relationale oder andere Ressourcen einzig im Interesse ihrer Mitglieder.

Im Gegensatz dazu sind barrierefreie Community Enterprises noch kaum systematisch untersucht worden. Sie produzieren Ressourcen, die niemand kontrolliert. Solche Ressourcen ermöglichen nachhaltige Innovation, weil sie Nachfolgeinnovatoren ohne Genehmigung zulassen. Zahlreiche Firmen, besonders in den schnell wachsenden Internet- und Informationstechnologieindustrien, nutzen diese Ressourcen. CEs kollidieren jedoch häufig mit profitorientierten Firmen, weil sie auf Prinzipien basieren, die einer konventionell verstandenen Marktwirtschaft fremd sind. Obwohl die Bedeutung von CEs vielfach anerkannt wird, tragen die Standardökonomik und die Theorie der Firma bisher wenig zur Erklärung ihres Erfolgs bei. Jedoch schaffen Community Enterprises große Werte - die allerdings mit herkömmlichen Methoden schwer messbar sind - welche es dringend notwendig erscheinen lassen, eine umfassende Theorie der Unternehmung und der Marktwirtschaft $\mathrm{zu}$ formulieren, welche neben konventionellen Firmen auch Community Enterprises zu erklären vermag. Davon sind wir derzeit noch weit entfernt.

Daraus ergeben sich zwei Konsequenzen für die Theoriebildung. Erstens müssen die Annahmen der ökonomischen Modelle daraufhin intensiv überprüft werden, ob sie die Erforschung neuer Phänomene wie der Community Enterprises und ihre Einpassung in ein breiteres Verständnis der Marktwirtschaft behindern. Zweitens sollte nach alternativen Maßen gesucht werden, welche die Aktivitäten und Resultate von CEs besser erfassen können. 